Also available at http://amc.imfm.si

ISSN 1855-3966 (printed edn.), ISSN 1855-3974 (electronic edn.)

ARS MATHEMATICA CONTEMPORANEA 3 (2010) 21-27

\title{
The generalized Reye configuration
}

\author{
Brigitte Servatius, Herman Servatius \\ Mathematics Department, Worcester Polytechnic Institute \\ 100 Institute Road, Worcester, MA 01609-2280, USA
}

Received 29 June 2009, accepted 29 November 2009, published online 28 January 2010

\begin{abstract}
Given an embedding in the plane of the vertices of a cube, we consider conditions on the embedded vertices such that they are the projection of the vertices of a 3 dimensional polyhedron combinatorially equivalent to the cube. We are particularly interested in conditions expressible as incidence conditions for the lines induced by the given vertex set in the plane. We show that the vertices and their incidences extend to a Reye configuration in the plane if and only if the vertices are the projection of a parallelepiped. More generally, the positions of the embedded vertices result from projecting a polyhedron combinatorially equivalent to the cube if and only if they belong to a plane configuration we call the generalized Reye configuration.
\end{abstract}

Keywords: Reye's configuration, generic picture, sharp lift.

Math. Subj. Class.: 51A05, 51A20, 51D20

\section{Introduction}

In [6], Theodor Reye addressed several questions concerning configurations and in [7] he examined what is now called Reye's configuration. An exposition of its properties is contained in [4, 5, 3] and a historical account is contained in [2]. For a modern application to quantum mechanics see [1].

A point-line configuration of type $\left(p_{n}, l_{m}\right)$ is a finite set of $p$ points and $l$ lines such that on each line there are $m$ points and through each point there are $n$ lines. Necessarily $p n=l m$. Similarly, one can consider configurations of points and planes. The Reye configuration is of type $\left(12_{4}, 16_{3}\right)$ and a realization in three dimensional real projective space may be obtained by taking the eight vertices of a cube together with the centroid of the cube and the three points at infinity of the three parallel classes of edges. The sixteen lines of the configuration are the twelve lines containing the edges of the cube together with the four main diagonals, see Figure 1. One can also consider the point-plane configuration

E-mail addresses: bservat@math.wpi.edu (Brigitte Servatius), hservat@wpi.edu (Herman Servatius) 
on the same set of points, with the planes determined by the six faces of the cube and the six planes determined by pairs of antipodal edges. The point-plane configuration is also known as Reye's configuration, and is of balanced type $\left(12_{6}, 12_{6}\right)$.

It is clear from the construction above that a realization of the point-line Reye configuration in the projective plane may be obtained from the projection of the vertices of a three dimensional cube, or more generally a parallelepiped, together with the centroid of the parallelepiped, which lies at the point of intersection of the four main diagonals, and three points at infinity corresponding to three parallel classes of lines, see Figure 1. Conversely, given a plane drawing of the points and lines of the 1-skeleton of a parallelepiped, we can ask if it must necessarily be the projection of a three dimensional parallelepiped. Clearly the main diagonals must be concurrent. Moreover, the four lines containing the edges corresponding to each perfect matching must be concurrent. In other words, the plane picture must be extendable to a Reye configuration. Is this condition sufficient? Would weaker conditions suffice?

Let us denote the combinatorial cube by $\mathcal{C}=(\mathcal{V}, \mathcal{E}, \mathcal{F})$. We will also use $\mathcal{C}$ to denote $(\mathcal{V}, \mathcal{E})$, the graph of the cube. A plane picture of $\mathcal{C}$ is an embedding $\pi(\mathcal{V})$ of $\mathcal{V}$ into the projective plane. The endpoints of edges of $\mathcal{C}$ determine a set of line segments between the vertices of the picture in the plane, which are allowed to cross. A lift of the picture $\pi$ is an embedding $\sigma(\mathcal{V})$ into 3 dimensional space such that $\sigma(\mathcal{V})$ projects (vertically) onto $\pi(\mathcal{V})$ such that any four points in $\sigma(\mathcal{V})$ corresponding to four vertices of a face $f \in \mathcal{F}$ must be coplanar. A lift is called sharp if distinct elements of $\mathcal{F}$ lift to distinct planes in space.

If a plane picture $\pi(\mathcal{C})$ may not be completed to a Reye configuration because of the failure of some required concurrence, $\pi(\mathcal{C})$ will not be the projection of any parallelepiped, but it may still have a sharp lift, see Figure $2 \mathrm{a}$.

On the other hand, it may happen that $\pi(\mathcal{V})$ has no sharp lift, see Figure $2 \mathrm{~b}$, where the dotted line indicates a crease that would be forced when a sharp lift of the five white vertices and the faces they span is extended to the last red vertex of $\mathcal{C}$. We would like to determine geometrically when a plane picture $\pi(\mathcal{C})$ has a sharp lift.

\section{Lifting $\pi(\mathcal{C})$}

In general, lifting a picture depends both on combinatorial constraints as well as the particular embedding. To study these aspects separately one defines a point set in the plane to be generic if perturbing the coordinates of any one point does not introduce any new lifts for pictures on that set. A sufficient condition for genericity is that the coordinates of the points are algebraically independent over the rationals. We know from [8] that a generic picture of the cube graph $\mathcal{C}$ has no sharp lift. Given a general incidence structure $(\mathcal{V}, \mathcal{P}, \mathcal{I})$ of vertices $\mathcal{V}$, planes $\mathcal{P}$ and incidences $\mathcal{I} \subseteq \mathcal{V} \times \mathcal{P}$, Whiteley [8] showed that a generic picture $\pi(\mathcal{V})$ has a sharp lift if and only if the inequality $|\mathcal{I}| \leq|\mathcal{V}|+3|\mathcal{P}|-4$ is satisfied for $(\mathcal{V}, \mathcal{P}, \mathcal{I})$ as well as all sub-incidence structures.

So a generic picture of $\mathcal{C}$ does not have a sharp lift. On the other hand, setting $\mathcal{V}=$ $\{A, B, C, D, E, F, G, H\}$ as in Figure 3 , if we remove one vertex, say $H$, as well as the three incidences containing $H$ from the incidence structure for $\mathcal{C}$, the resulting incidence structure does satisfy Whiteley's condition, and so do all its sub-incidence structures. Hence a generic picture of $\pi(\mathcal{C}-H)$ has a sharp lift into 3-space. Moreover, since $\pi(\mathcal{C}-H)$ is generic, the three planes corresponding to the three faces incident to $H$ intersect in one point, call it $\sigma(H)$, in space, which projects back to a point $\pi(H)$ in the plane, extending 


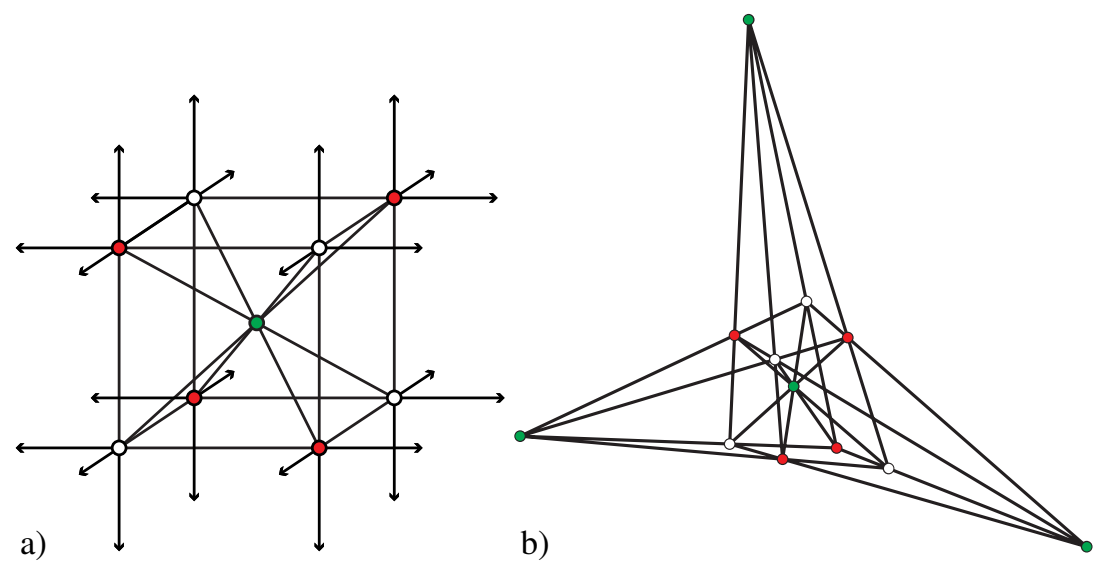

Figure 1: Two views of the Reye configuration. The figure on the left shows the eight red and white "cube" vertices and the green "centroid" vertex, with the three points at infinity indicated by the arrows. The figure on the right is foreshortened with all finite points, and, projectively, vertices of any two colors could play the role of the cube vertices, and any vertex of the remaining color could play the role of the centroid.

a)

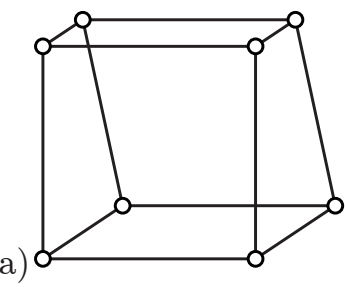

b)

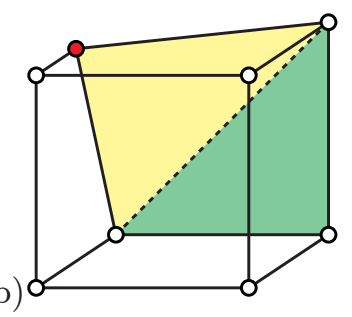

Figure 2: The picture of the cube graph on the left is liftable to a trapezoidal prism. By contrast the picture on the right has no lift, and the dotted line indicates a forced crease.

$\pi(\mathcal{C}-H)$ non-generically to a liftable picture $\pi(\mathcal{C})$. Note that the same conclusion holds if we assume only that the three quadrilaterals of $\pi(\mathcal{C}-H)$ are each in general position.

Given a sharp lift of $\pi(\mathcal{C}-H)$, the planes through $\{\sigma(A), \sigma(B), \sigma(C), \sigma(D)\}$ and $\{\sigma(E), \sigma(F), \sigma(G)\}$ intersect in a line in space which projects to a line in the plane determined by the intersection point of lines $\pi(A) \pi(B)$ and $\pi(E) \pi(F)$, call it $\pi(P)$, as well as the intersection point of lines $\pi(B) \pi(C)$ and $\pi(F) \pi(G)$, call it $\pi(Q)$, see Figure 3. Let $\pi(A) \pi(D)$ intersect $\pi(P) \pi(Q)$ at $\pi(S)$, so $\pi(H)$ must lie on $\pi(S) \pi(E)$. By the same token, $\pi(H)$ must lie on $\pi(R) \pi(G)$. Note that sharp lifts of $\pi(\mathcal{C}-H)$ give rise to precisely the same point $\pi(H)$.

Theorem 2.1. Given the cube graph $\mathcal{C}$ with vertex set $\mathcal{V}=\{A, \ldots, H\}$ and given seven generic points $\{\pi(A), \ldots, \pi(G)\}$ in the plane, there is a unique eighth point $\pi(H)$ so that $\pi(\mathcal{C})$ is a plane picture of $\mathcal{C}$ with a sharp lift.

If the picture of $\mathcal{C}$ has a sharp lift, then the quadrilaterals corresponding to a pair of opposite faces in $\mathcal{C}$ are perspective from a line. On the other hand, we have just seen that 


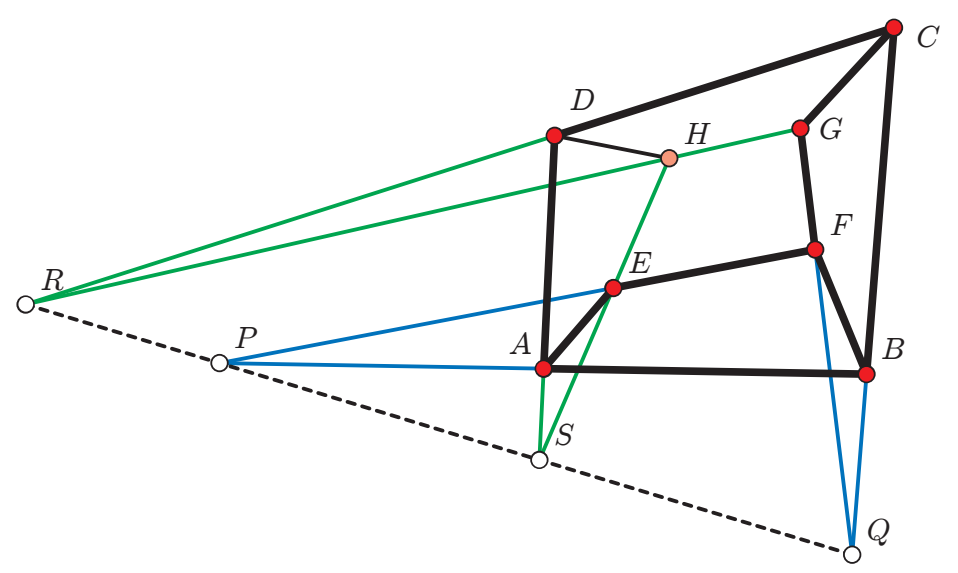

Figure 3: A purely planar construction of $\pi(H)$ from $\pi(\mathcal{C}-H)$ : If there is a lift, then the line joining $P=(E F \wedge A B)$ and $Q=(B C \wedge F G)$ must contain the points $S=$ $(A D \wedge E H)$ and $R=(C D \wedge G H)$.

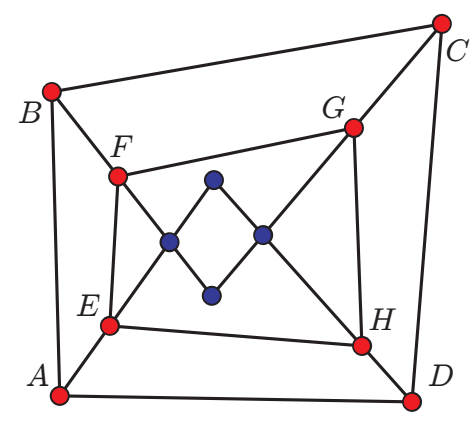

Figure 4: Corresponding pairs of points belonging to opposite quadrilaterals $A B C D$ and $E F G H$ induce lines intersecting at the four blue vertices.

if the quadrilaterals corresponding to any pair of opposite sides in $\mathcal{C}$ are perspective from a line, then that picture is the unique picture corresponding to the sharp lift guaranteed by the incidences. So we have the following.

Theorem 2.2. Given a picture $\pi(\mathcal{C})$ of $\mathcal{C}=(\mathcal{V}, \mathcal{E}, \mathcal{F})$ such that the points $\pi(f)$ are in general position for each $f \in \mathcal{F}$. If two quadrilaterals corresponding to a pair of opposite faces in $\mathcal{C}$ are perspective from a line, then all pairs of quadrilaterals in $\pi(\mathcal{C})$ corresponding to a pair of opposite faces in $\mathcal{C}$ are perspective from a line.

\section{The generalized Reye configuration}

We call a picture $\pi(\mathcal{C})$ of $\mathcal{C}=(\mathcal{V}, \mathcal{E}, \mathcal{F})$ a general picture if the points $\pi(f)$ are in general position for each $f \in \mathcal{F}$. Suppose that we have a general picture $\pi(\mathcal{C})$ of $\mathcal{C}$ in which some, and hence all, pairs of opposite quadrilaterals are perspective from a line. So the picture lifts to a spatial picture $\sigma(\mathcal{C})$. If each of the three pairs of opposite quadrilaterals are also perspective from a point, perhaps at infinity, then the spatial and plane pictures 


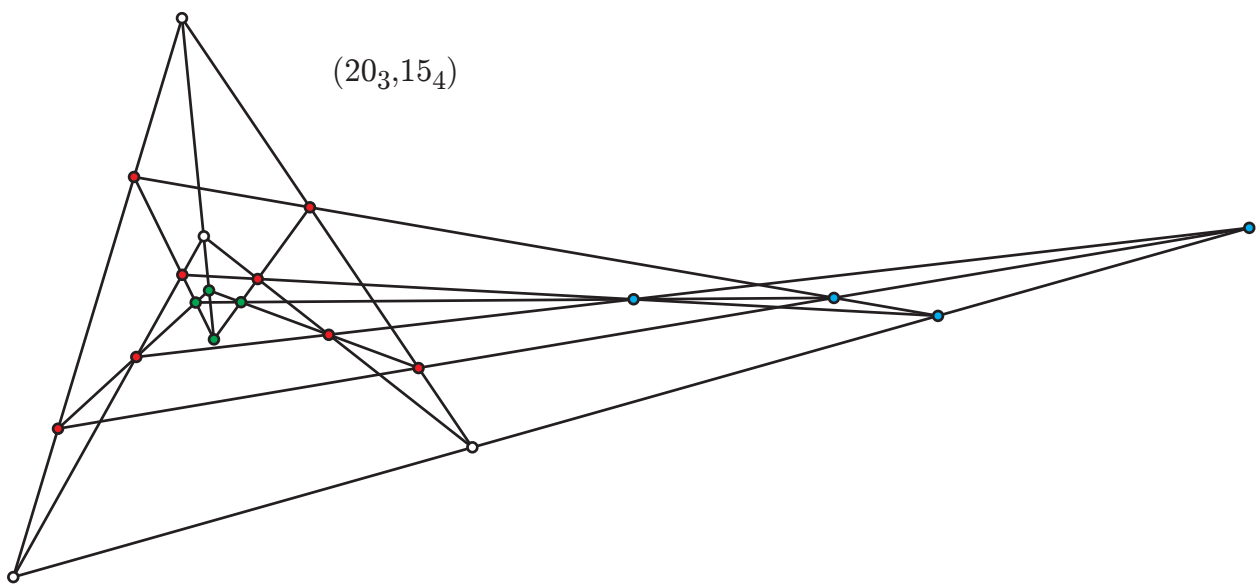

Figure 5: The generalized Reye configuration in which the vertices of the cube graph are red, and the 4-sets of intersection points of the lines connecting corresponding points of opposite quadrilaterals are colored white, blue and green.

can be completed to Reye configurations. If this is not the case, then the pairs of opposite quadrilaterals each give rise to up to four intersection points, see Figure 4 . Note that the lines $\sigma(A) \sigma(E)$ and $\sigma(C) \sigma(G)$ will in general be skew in space, and so need not intersect, similarly for the lines $\sigma(B) \sigma(F)$ and $\sigma(D) \sigma(H)$. The line of intersection of the opposite quadrilaterals $(\sigma(A) \sigma(D) \sigma(H) \sigma(E))$ and $(\sigma(B) \sigma(C) \sigma(G) \sigma(F))$ contains the point $\sigma(A) \sigma(E) \wedge \sigma(B) \sigma(F)$, as well as $\sigma(C) \sigma(G) \wedge \sigma(D) \sigma(H), \sigma(B) \sigma(C) \wedge \sigma(A) \sigma(D)$ and $\sigma(F) \sigma(G) \wedge \sigma(E) \sigma(H)$. So we may add the three sets of four intersection points corresponding to the opposite quadrilaterals, and this set of twelve new points is partitioned by the three lines of perspectivity of the opposite quadrilaterals. Adding these three lines as well gives us a configuration of twenty points and fifteen lines of type $\left(20_{3}, 15_{4}\right)$ which we call the generalized Reye configuration, see Figure 5.

In analogy with the relationship between the Reye configuration and the parallelepiped, we may say the following:

Theorem 3.1. A general picture $\pi(\mathcal{C})$ of the cube graph has a sharp lift if and only if it extends to a generalized Reye configuration.

For a plane picture of a triangular prism, Desargues' Theorem provides an equivalence between the triangular faces being perspective from a point and perspective from a line. There is no such symmetry for the plane picture of $\mathcal{C}$. It is not true that if some pair of opposite quadrilaterals is perspective from a point then all three pairs are. In Figure 6a

we have a configuration in which all three pairs of opposite quadrilaterals are perspective from a line, so it has a lift, and the vertices of two pairs of opposite quadrilaterals are perspective from points, but the third, shaded, pair is not in perspective from a point.

However, we can say the following.

Theorem 3.2. If two pairs of opposite quadrilaterals in a general picture $\pi(\mathcal{C})$ of $\mathcal{C}$ are in perspective from points, then $\pi(\mathcal{C})$ has a lift. 

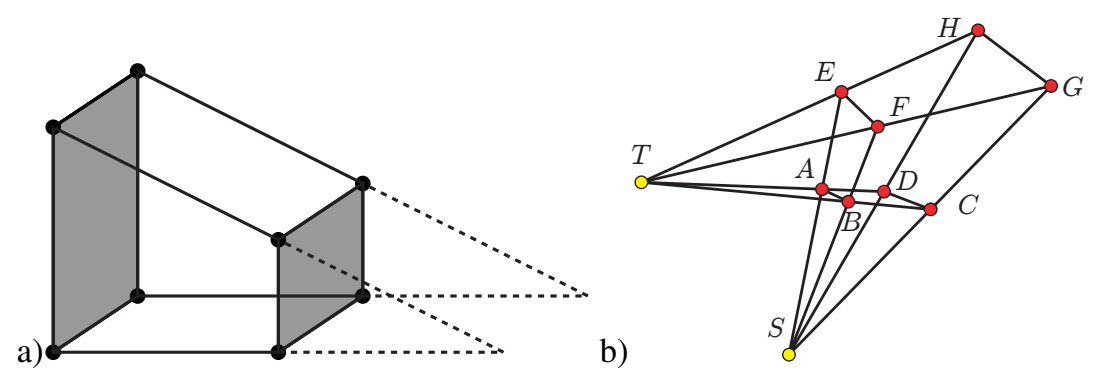

Figure 6: On the left only two of the three pairs of opposite quadrilaterals are perspective from a point. On the right, two pairs of opposite quadrilaterals perspective from a point implies the third pair is perspective from the line joining them.

a) $\quad l=3 \quad p=3$
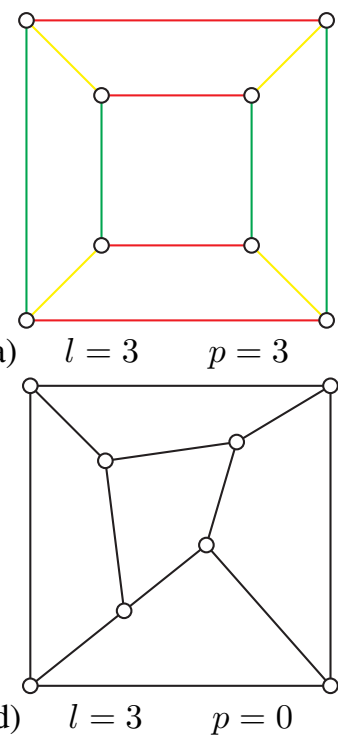

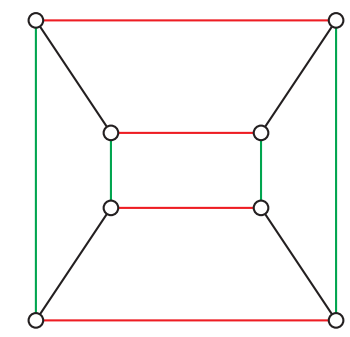

b) $\quad l=3 \quad p=2$

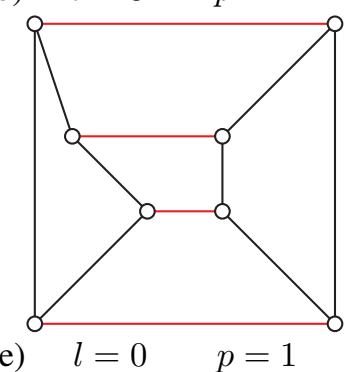

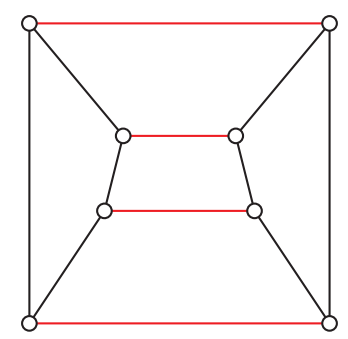

c) $\quad l=3 \quad p=1$

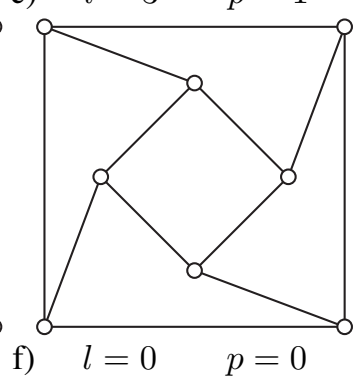

Figure 7: Examples of pictures of a cube graph classified by liftability and perspectivity of opposite pairs of quadrilaterals. Colored matchings in each figure are perspective from a point.

Proof. If two pairs of opposite quadrilaterals in a plane picture $\pi(\mathcal{C})$ of $\mathcal{C}$ are in perspective from two points $S$ and $T$, then the third pair of opposite quadrilaterals has only $S$ and $T$ as points of perspectivity, see Figure $6 \mathrm{~b}$, so the third pair of opposite quadrilaterals is perspective from the line $S T$, hence $\pi(\mathcal{C})$ has a lift.

There are six cases for general pictures $\pi(\mathcal{C})$ of the cube graph distinguished by the number of pairs of opposite quadrilaterals which are perspective from a line $l$ and the number which are perspective from a point $p$, see Figure $7 \mathrm{a}$.

The generalized Reye configuration has been defined as a point-line configuration, but Theorem 3.1 implies that it may also be viewed as point-plane configuration of type $\left(20_{3}, 6_{10}\right)$. 


\section{References}

[1] P. K. Aravind, Reye's configuration helps in proving the Bell-Kochen-Specker theorem: a curious geometrical tale, Found. Phys. Lett. 13 (2000), 499-519.

[2] H. Gropp, Die Configurationen von Theodor Reye in Straßburg nach 1876, In Mathematik im Wandel, volume 3 of Math. gesch. Unterr., Franzbecker, Hildesheim, 2001, 287-301.

[3] D. Hilbert and S. Cohn-Vossen, Geometry and the imagination, Chelsea Publishing Company, New York, N. Y., 1952, Translated by P. Neményi.

[4] D. Hilbert and S. Cohn-Vossen, Anschauliche Geometrie, Wissenschaftliche Buchgesellschaft, Darmstadt, 1973.

[5] D. Hilbert and S. Cohn-Vossen, Naglyadnaya geometriya, third edition, Nauka, Moscow, 1981, Translated from the German by S. A. Kamenetskiu.

[6] T. Reye, Das Problem der Configurationen, Acta Math. 1 (1882), 93-96.

[7] T. Reye, Die Hexaëder- und die Octaëder-Configurationen $\left(12^{6}, 16^{3}\right)$, Acta Math. 1 (1882), 97 108.

[8] W. Whiteley, A matroid on hypergraphs, with applications in scene analysis and geometry, Discrete Comput. Geom. 4 (1989), 75-95. 